\title{
A METHOD FOR RAPID VULNERABILITY ASSESSMENT OF STRUCTURES LOADED BY OUTSIDE BLASTS
}

\author{
Leon Cizelj ${ }^{1}$, Matjaž Leskovar, Marko Čepin, Borut Mavko \\ “Jožef Stefan” Institute, Reactor Engineering Division
}

\section{Keywords}

blast loads, buildings, rapid assessment, structural reliability

\section{ABSTRACT}

The blast loads have in most cases not been assumed as design basis loads of nuclear power plant buildings and structures. Recent developments however stimulated a number of analyses quantifying the potential effects of such loads.

An effort was therefore made by the authors to revisit simple and robust structural analysis methods and to propose their use in the vulnerability assessment of blast-loaded structures. The leading idea is to break the structure into a set of typical structural elements, for which the response is estimated by the use of slightly modified handbook formulas. The proposed method includes provisions to predict the inelastic response and failure. Simplicity and versatility of the method facilitate its use in structural reliability calculations.

The most important aspects of the proposed method are presented along with illustrative sample applications demonstrating:

- results comparable to full scale dynamic simulations using explicit finite element codes and

- the performance of the method in screening the existing structures and providing the structural reliability information for the vulnerability analysis.

$\begin{array}{lccccc} & \text { Corresponding } \quad \text { Author: Jamova } 39, \quad \text { SI-1000 Ljubljana, } & \text { Slovenia }\end{array}$




\section{INTRODUCTION}

Significant worldwide efforts have been recently devoted to the increased security of nuclear power plants (NPP) (see for example Čepin et al, 2002). In particular, potential vulnerability to the external events has been revisited and analyzed in greater detail. The first studies of the kind scrutinized the experience from military threats to a nuclear power plant in the year 1991 (Stritar et al, 1991). More recent examples investigate threats posed by the crash of commercial aircrafts (Jovall, 2007) or by the explosion of a vehicle bomb (Čepin et al, 2006).

A vulnerability analysis of a complex industrial facility such as for example nuclear power plant requires a rather broad scope of investigations. As an example, slightly modified PSA/PRA methods may be successfully used to analyze the consequences of a potentially successful threat. An important part of input to the consequence analysis is assessment of potential damage to the buildings and the equipment housed in the potentially damaged buildings.

An effort was therefore made by the authors to revisit simple and robust structural analysis methods and to propose their use in the vulnerability assessment of blast-loaded structures. For this purpose, the structure is broken down into a set of typical structural elements, for which the response is estimated by the use of slightly modified handbook formulas. The proposed method includes basic provisions to predict the inelastic response and failure. Simplicity and versatility of the method facilitate its use in structural reliability calculations.

Complex simulations of interactions between blast, buildings and internal structures/systems are beyond the scope of this paper. 


\section{MODEL}

The basic loading imposed on the structures by the remote explosions is the blast with approximately spherical pressure waves. The dominant damage effects considered in his paper are attributed to the positive overpressure. The negative pressure pulse, possible focusing of the blast, fragments generated by the explosive device etc. are outside the scope of this paper.

The main parameters describing the blast loading are the sudden peak overpressure $p_{\max }$ and the decay the overpressure phase. Two decay forms are of particular interest here: exponential, which is well known to accurately approximate the observed decay:

$$
p(t)=p_{\max } \cdot \exp \left(-\frac{2 t}{t_{d}}\right) \Theta(t)
$$

and linear, which is very convenient for fast assessments:

$$
p(t)=p_{\max } \cdot\left(1-\frac{t}{t_{d}}\right) \Theta(t) \Theta\left(t-t_{d}\right) .
$$

$t$ represents time, $t_{d}$ the duration of the overpressure pulse, and $\Theta$ the Heaviside theta function. Both decay curves (Figure 1) have been chosen to result in identical pressure impulses $I$ :

$$
I=\int p(t) d t=\frac{1}{2} \cdot p_{\max } \cdot t_{d} .
$$

The distance between the bomb and the target is assumed to be large enough to develop the pressure waves, which are essentially parallel to the target wall. Such an assumption seems to be conservative also for bombs closer to the target, of course taking into account the much stronger reflection of the pressure wave due to the vicinity of the bomb in accordance with the models applied in (WST 3.4).

The overpressure reflected from the wall parallel to the overpressure wave is conservatively assumed in all calculations, as obtained from (WST 3.4). Other incident angles are not taken into account since they result in lower blast loadings to the structure (Smith \& Hetherington, 1994).

\subsection{Model of a building}

The blast loading on the buildings is to the first approximation taken by the vertical walls, representing the vast majority of the building outside surface. The vertical walls are typically supported with internal walls, columns, podiums, etc. With respect to an outside blast, the stiffness of such supports is much higher than the stiffness of the wall. 
A reasonable representative model for a building is therefore a set of wall segments. For the simplicity, a homogeneous isotropic rectangular plate clamped at all four sides is chosen to represent each wall segment. The size of the plate is defined with the distance between supports.

The first natural frequency f or period $T$ of the clamped rectangular plate of width $a$, length $b$ and thickness $h$ is then defined as (Harris \& Crede, 1976):

$$
f=\frac{1}{T}=B_{a} \sqrt{\frac{E h^{2}}{2 \pi 12\left(1-v^{2}\right) \rho a^{4}}} .
$$

$B_{a}$ is shape factor, outlined in Table $1, E$ Young's modulus, $v$ Poisson ratio and $\rho$ density. The maximum static deflection of the plate loaded with uniform pressure $p$ is further estimated as (Beitz \& Küttner, 1981):

$$
w_{\max }=C_{3} \frac{p}{E h^{2}}\left(\frac{a}{2}\right)^{4} .
$$

Maximal stresses at the center and clamped border of the plate are given as (Beitz \& Küttner, 1981):

$$
\begin{aligned}
& \sigma_{\text {center }}=C_{2} \frac{p}{h^{2}}\left(\frac{a}{2}\right)^{2} \text { and } \\
& \sigma_{\text {border }}=C_{5} \frac{p}{h^{2}}\left(\frac{a}{2}\right)^{2} .
\end{aligned}
$$

Shape factors $C_{2}, C_{3}$ and $C_{5}$ are given in Table 1 . It is clear from Table 1 that the maximum stress in the plate will always occur close to the middle of the longest clamped side. Additionally, plate with side length aspect ratio of about 2 seems to be a reasonably representative choice for most practical purposes.

\subsection{Equivalent 1-D oscilator}

It is customary to assume that an equivalent 1-D oscillator provides reasonable approximation of the motion (Smith \& Hetherington, 1994) of the plate due to the blast loading. Neglecting the damping, the 1-D oscillator is defined as:

$$
m_{E} \ddot{x}+k_{E} x=F_{E}(t) .
$$

$m_{E}$ an $k_{E}$ represent the equivalent mass and equivalent stiffness of the system and $F_{E}(t)$ the equivalent forcing function. $x$ and $\ddot{x}$ represent displacement and acceleration, respectively.

Parametric description of pressure decay curves in eqs. (1) and (2) allows parameterization of the equivalent forcing function: 


$$
F_{E}(t) \propto p(t) \Rightarrow F_{E, \max } \propto p_{\max } .
$$

Solution of equation (8) is now, assuming zero initial displacement and velocity, straightforward. For exponential decay (eq. (1)) it follows that:

$$
x(t)=\frac{F_{E, \max } t_{d}{ }^{2}}{4 m_{E}+k_{E} t_{d}{ }^{2}}\left(e^{\frac{-2 t}{t_{d}}}-\cos (\omega t)+\frac{2}{\omega t_{d}} \sin (\omega t)\right) .
$$

Similarly, for linear decay (eq. (2)):

$$
x(t)=\frac{F_{E, \max }}{k_{E} t_{d}}\left(t_{d}(1-\cos (\omega t)-t)+\frac{F_{E, \max } \omega}{k_{E} t_{d}} \sin (\omega t)\right.
$$

for $t<t_{d}$ and

$$
x(t)=\frac{F_{E, \max } \omega}{k_{E} t_{d}}\left(\sin \left(\omega\left(t-t_{d}\right)\right)-\sin (\omega t)\right)-\frac{F_{E, \max }}{k_{E}} \cos (\omega t)
$$

for $t>t_{d}$. Note that $\omega=\sqrt{ }\left(k_{E} / m_{E}\right)$.

Equivalence of the 1-D oscillator requires equality of the kinetic and deformation energy and the work of the external forces of the clamped plate and the oscillator. The kinetic $E_{K}$ and deformation energy $E_{D}$ of the plate are given as:

$$
\begin{aligned}
& E_{K}=\frac{1}{2} \rho a b h \int_{0}^{b} \int_{0}^{a}\left(\frac{\partial w(x, y)}{\partial t}\right)^{2} d x d y \text { and } \\
& E_{D}=\frac{1}{2} \frac{E h^{3}}{12\left(1-v^{2}\right)}\left(\int_{0}^{b} \int_{0}^{a}\left(\frac{\partial^{2} w(x, y)}{\partial x^{2}}+\frac{\partial^{2} w(x, y)}{\partial y^{2}}\right)^{2} d x d y-\right. \\
& \left.-2(1-v) \int_{0}^{b} \int_{0}^{a} \frac{\partial^{2} w(x, y)}{\partial x^{2}} \frac{\partial^{2} w(x, y)}{\partial y^{2}}-\left(\frac{\partial^{2} w(x, y)}{\partial x \partial y}\right)^{2} d x d y\right)
\end{aligned}
$$

The work of the external forces $E_{F}$ is given as:

$$
E_{F}=p a b \int_{0}^{b} \int_{0}^{a} w(x, y) d x d y .
$$

In order to estimate the deformation and kinetic energies of the deformed plate, it is useful to assume the distribution of the plate deflections in a simple form, satisfying the clamped boundary conditions. Two rather obvious examples resulting in closed form solutions for plate energy are polynomial:

$$
w(x, y)=w_{\max } \cdot \frac{16(a-x)^{2} x^{2}}{a^{4}} \cdot \frac{16(b-y)^{2} y^{2}}{b^{4}}
$$

and cosine approximation: 


$$
w(x, y)=w_{\max } \cdot \frac{1}{4}\left(\cos \left(2 \frac{x}{a} \pi\right)-1\right)\left(\cos \left(2 \frac{y}{b} \pi\right)-1\right) .
$$

The polynomial distribution is depicted in Figure 2.

Now, the parameters of the equivalent oscillator are given as

$$
\begin{aligned}
& m_{E}=\rho a b h\left\{\begin{array}{l}
0.165, \text { polynom } \\
0.141, \text { cosinus }
\end{array},\right. \\
& k_{E}=c_{3} \frac{p a b}{w_{\max }}\left\{\begin{array}{c}
0.068 \frac{7 a^{4}+4 a^{2} b^{2}+7 b^{2}}{b^{4}}, \text { polynom } \\
0.139 \frac{3 a^{4}+2 a^{2} b^{2}+3 b^{2}}{b^{4}}, \text { cosinus }
\end{array}\right. \text { and } \\
& F_{E, \max }=p_{\max } a b\left\{\begin{array}{c}
0.284, \text { polynomial } \\
0.25, \text { cosinus }
\end{array} .\right.
\end{aligned}
$$

The difference between both distributions is expected to be rather small (Smith \& Hetherington, 1994). It is shown above that the equivalence factors derived by both assumptions vary for about 10 to $15 \%$. This is also the maximum error expected in the estimated plate energies. The cosinus distribution of deflections stores slightly less energy in the plate and therefore makes the plate relatively more vulnerable as the polynomial distribution.

\subsection{Limits of the equivalent 1-D oscillator}

The dynamic response depends heavily on the duration of the overpressure phase $t_{d}$ as compared to the period of the structure $T$. Two limiting cases exist:

- The period longer than overpressure phase $\left(T>2,5 t_{d}\right)$. The impulsive loading prevails leading to the balance of kinetic $E_{K}$ and deformation energy $E_{D}$ of the structure. This in turn leads to the maximum dynamic deflection of the (elastic) plate $w_{D}$ :

$$
w_{D}=\frac{1}{2} \omega t_{d} \frac{F_{E}}{k_{E}} \text {. }
$$

- The period shorter than overpressure (40 $T<t_{d}$ ). The loading on the plate is safely assumed to be quasi static. The quasi static maximum plate deflection then follows directly from the stiffness and external loading of the plate, taking into account the well known dynamic load factor of 2 (Smith \& Hetherington, 1994):

$$
w_{Q S}=2 \frac{F_{E}}{k_{E}} \text {. }
$$




\subsection{Damage estimates}

Basically, the equations above assume that the load carrying capacity of the wall is characterized by the strain energy and is estimated using available elastic solutions for (clamped) plates. The plastic or irreversible deformation energy is accounted for using the ductility ratio $m$ (Mays \& Smith, 1995) between the actual deformation energy stored in the wall and the maximum elastic deformation energy stored at the on-set of yielding or initiation of damage.

The yield strength $\sigma_{Y}$ is taken here as a measure of the deformation energy which can be stored in the material in a reversible manner, i.e., without yielding or damage. Eqs. (6) and (7) at the limit of $b / a \rightarrow \infty$ then lead to the maximum reversible deflection of the wall:

$$
w_{E L}=C_{3} \frac{\sigma_{Y}}{E} \frac{a^{2}}{8} .
$$

The ductility ratio of the given load is then estimated using:

$$
m=\frac{w_{D}}{w_{E L}}
$$

for the dynamic and

$$
m=\frac{w_{Q S}}{w_{E L}}
$$

for the quasi static loads.

Three qualitative levels of damage possibly experienced by the building are then estimated as:

- $\quad$ no damage - purely reversible response with $m \leq 1$;

- wall collapse denotes a severe damage of the wall, described by wall collapse of wall destruction. It is assumed to occur when the strain energy in the plate exceeds the reversible strain energy by a factor of $m_{C}\left(m>m_{C}\right)$;

- destroy systems and/or structures in the first compartment immediately after the collapsed wall. Here it is assumed that at least the amount of blast energy needed for wall collapse shall remain available to destroy the systems in the first compartment behind the collapsed/destroyed wall $\left(m>2 m_{C}\right)$.

The selection of values for $\sigma_{Y}$ and $m_{C}$ is crucial for the validity of the procedure and may be based for example on engineering judgment, a detailed analysis of a very complex material behavior and last, but not least, on experimental data. As a very rough guide, ductile structural steel with yield strength of $\sigma_{Y}$ will be reasonably described with $m_{C}$ of 20 . For 
reinforced concrete structures with compressive strength equal to $\sigma_{Y}$, value of 2-3 may be appropriate. Alternatively: if $\sigma_{Y}$ is chosen to represent the tensile strength of the concrete (typically about $10 \%$ of the compressive strength), $m_{C}$ of 20 could be a reasonable choice representing reinforced concrete. 


\section{COMPARISON WITH FINITE ELEMENT SOLUTIONS}

A sensitivity analysis has been performed using explicit finite element code ABAQUS/Explicit (ABAQUS 6.5.1). The finite element results were compared with the results obtained by the 1-D equivalent oscillator. The sensitivity analysis included the following variations of the input data:

- Geometry: square and rectangular plate with dimensions 5 by 5 by $0,1 \mathrm{~m}$ and 10 by 5 by $0,1 \mathrm{~m}$ were analyzed. The finite element consisted of linear 3-D elements of typical size of about 0,25 by 0,25 by $0,05 \mathrm{~m}$.

- Loading: The peak overpressure $p_{\max }$ was held constant at 1 bar $(0,1 \mathrm{MPa})$. The overpressure duration $t_{d}$ was varied as a function of the first natural period as indicated in Table 2. Both exponential and linear pressure decay (eqs. (1) and (2), respectively) were used in the analyses.

- Material: A homogeneous and isotropic approximation of the reinforced concrete was assumed with $\rho=3000 \mathrm{~kg} / \mathrm{m} 3, E=15 \mathrm{GPa}$ and $v=0,2$. Both elastic and elastic-ideally plastic analyses with yield strength of $5 \mathrm{MPa}$ were performed.

As a general rule, the linear decay of pressure (eq. (2)) was found to consistently produce slightly larger deflection than the exponential (eq. (1)). This is noted in both 1-D and finite element solution and is attributed to the conservative nature of the linear decay, which delivers the pressure impulse to the structure in shorter time as compared to the exponential decay. The discussion of the results below is therefore limited to those obtained using linear pressure decay (eq. (2)) in both finite element model and equivalent oscillator. Also, as already noted in section 2.3 , the cosine approximation of the plate deflection is deemed representative for the equivalent oscillator.

Comparison of maximum plate deflections estimated by the equivalent oscillator with elastic and elastic-plastic finite element calculations are depicted in Figure 3, Figure 4 and Figure 5. Very good agreement of all compared models is shown for short overpressure phases in Figure 3 and Figure 4. Rather short overpressure phases result in relatively small deflections, and consequently, in negligible influence of plastic deformations, which are localized within the pate. The suggested limiting value of dynamic deflection, given in eq. (21) is clearly a valid limit also for all models analyzed in this paper. It is concluded that at short overpressure phases both equivalent oscillator and finite element model give very comparable results. The 1-D equivalent oscillator is therefore concluded to be an accurate, reliable and very robust method. 
For longer overpressure phases, the results of the equivalent oscillator and the finite element solutions differ considerably (Figure 5). Rather long duration of the overpressure caused significant increase in the maximum plate deflection amplitude. This in turn caused significant amount of plastic deformation, which is basically shown as significant reduction of the amplitude after the first oscillation of the plate. Nevertheless, the maximum amplitude of both finite element models is clearly overestimated by the 1-D oscillator, which tends to obey the quasystatic limit suggested in eq. (22). The equivalent oscillator is concluded to be conservative here. As a consequence, the vulnerability estimates of the equivalent estimators may be considered unrealistically severe. Detailed analyses should be given priority in such cases.

The 1-D equivalent oscillator is therefore shown to be a simple, reliable and robust tool for fast assessment of impulsively blast-loaded plates. 


\section{VULNERABILITY ANALYSIS}

The above structural assessment method has been implemented in the vulnerability analysis of a generic and simplified industrial facility (e.g., nuclear power plant) to a deliberately caused damage (see Figure 6). The particular damage source studied was an explosion of a device brought to the location by land transport. A broad sensitivity study varying the amount and position of the explosive has been conducted to assess the vulnerability of the facility and to set up the basis for an effective protection (Čepin et al, 2006).

The results shown hereafter are intended to be illustrative and therefore correct in the qualitative but not always quantitative sense to avoid possible misuse. Quantitative values of the selected resulting parameters are deliberately not shown to scale neither in Figures nor in the text.

\subsection{Buildings and systems fragility}

In the first step it is necessary to identify:

- plant buildings, their location and their blast response properties,

- systems important to safety and their location. It is especially important to locate the systems, which are at least in part located in the first compartments behind the outside wall.

The buildings in a typical NPP with Pressurized Water Reactor (PWR), which are important for the technological processes and plant safety, may be divided in two broad categories:

- Reinforced concrete buildings with walls made of reinforced concrete.

- Steel frame buildings with metal sheet walls.

As the blast loading decreases with the distance from the explosion, it is possible to estimate the stand-off distances for each type of the buildings analyzed (see Figure 7). The simple structural models described in section 2 have been used to estimate the stand of distances for "no damage", "wall collapse" and "systems destroyed". In other words, given the properties of the wall and the location, mass and type of the explosive device, we may use the above simplified models to predict the level of damage on the structure and systems contained in the structure. 
The component and system fragility analysis takes place only in buildings or behind the walls with damage levels "wall collapse" and/or "destroy systems". For the buildings with damage level "wall collapse", immediate failure was assumed for the equipment, which is:

- mounted on the collapsed wall;

- sensitive to the air pressure or on change of air pressure in its vicinity.

For the buildings with damage level "destroy systems", immediate failure was assumed for all equipment in the first compartment behind the damaged wall.

\subsection{Results}

Figure 8 shows the stand-off distances for a selected bomb placed inside the controlled area but outside of the technological buildings. Each stand off distance divides the area around the target in a distant safe and a near unsafe zone.

The dashed thin and thick black lines show the "no damage" and "collapse" stand-off distances, which are typical for the steel frame building. The solid thin and thick black lines denote the "no damage" and "collapse" stand-off distances for reinforced concrete plates of different wall thicknesses. All stand-off distances are plotted around respective buildings.

The stand-off distances for human injuries were determined and plotted around the centre of the office. The dashed and solid gray lines denote the distances for eardrum rupture probability $50 \%$ and lung damage death probability $50 \%$ inside the office if the vehicle bomb would be at the location of the corresponding gray contour line. It was assumed that the blast parameters in the office are like they would be in a free-air hemisphere without any obstacles. This is probably much too conservative, since the office is located quite inside the building and a blast entering from a "free field" into branched channels rapidly looses its power. Therefore the gray human injury contour lines should be regarded only as very conservative bounds. They are presented mainly to give us an impression about human injury damage distances in free air, and therefore they are plotted also inside the buildings, where a vehicle bomb could not be located. It is however worth noting that injuries and casualties among the facility staff are likely to affect the performance of the plant operators (if the example facility would be a NPP).

The stand-off distances for system failure were determined according to the wall thickness of the concrete buildings inside which the systems are located, using the calculated damage distances. The stand-off distances for system failure are denoted with the extra thick black lines, which are plotted near the concrete building walls. If the bomb would be located 
between the stand-off distances for system failure and the building, the system inside the building would be destroyed.

The stand-off distances were plotted in such a way that it is possible to establish to which part of the building they belong (the contour lines were not cut off at their intersections). 


\section{CONCLUSIONS}

Simple and robust structural analysis methods have been revisited and proposed for use in the vulnerability assessment of blast-loaded structures. The leading idea was to break the structure into a set of typical structural elements, for which the response is estimated by the use of slightly modified handbook formulas. The proposed method includes basic provisions to predict the inelastic response and failure.

The most important aspects of the proposed method are presented along with illustrative sample applications demonstrating results comparable to full scale dynamic simulations using explicit finite element codes.

The performance of the method is illustrated in the vulnerability analysis of a generic and simplified industrial facility, e.g., nuclear power plant, to a deliberately caused damage. Simplicity and versatility of the method facilitate its use in structural reliability calculations. 


\section{REFERENCES}

W. Beizz, K.-H. Küttner, Dubbel Taschenbuch für den Maschinenbau (14. Auflage), Springer 1981.

M. Čepin, L. Cizelj, L. Fabjan, B. Mavko, Assessment of External Events (in Slovenian), IJSDP-8648, IJS, Ljubljana, November 2002.

M. Čepin, L. Cizelj, M. Leskovar, B.Mavko, Vulnerability Analysis of a Nuclear Power Plant Considering Detonations of Explosive Devices, Journal of Nuclear Science and Technology, Vol. 43 2006, pp. 1258-1269.

O. Jovall, Airplane Crash Simulations, Proceeding of the 15 International conference on Nuclear Engineering ICONE-15, Nagoya, Japan, April 2007, Paper No. 10474.

C.M. Harris, C.E.Crede (eds.), Schock \& Vibration Handbook (2nd Ed.), McGraw-Hill, New York, 1976.

Hibbit, Karlsson and Sorrensen Inc., ABAQUS/Explicit 6.5.1, www.abaqus.com

G.C.Mays, P.D.Smith (eds.), Blast Effects on Buildings, Thomas Telford Publications, London, UK, 1995.

P.D. Smith, J.G. Hetherington, Blast and Ballistic Loading of Structures, ButterworthHeineman Ltd., Oxford, Great Britain, 1994.

A. Stritar, B. Mavko, J. Sušnik, B. Šarler, Some Aspects of Nuclear Power Plant Safety Under War Conditions, Nuclear Technology, Vol. 101, Februar 1993, pp. 193-201.

WST 3.4, Weapon Safety Tools Version 3.4, Program for Windows environment, http://www.hill.af.mil/safety/weapframe.htm. 


\section{LIST OF FIGURES}

Figure 1 Pressure decay curves (blue/full - exponential, red/dotted - linear)

Figure 2 Deflections of the (square) plate

Figure 3 Deflections of the rectangular plate (very short overpressure phase $t_{d}=0,01 T$ )

Figure 4 Deflections of the rectangular plate (short overpressure phase $t_{d}=0,1 T$ )

Figure 5 Deflections of the rectangular plate (long overpressure phase $t_{d}=10 \mathrm{~T}$ )

Figure 6 Ground Plan of the Example Facility with Building

Figure 7 Stand-off distances around a selected bomb. The distances are not to scale

Figure 8 Stand-off distances for a selected bomb inside the controlled area. The distances are not to scale

\section{LIST OF TABLES}

Table 1 Shape factors (Harris \& Crede, 1976), (Beitz \& Küttner, 1981)

Table 2 Periods and overpressure durations 


\section{FIGURES}

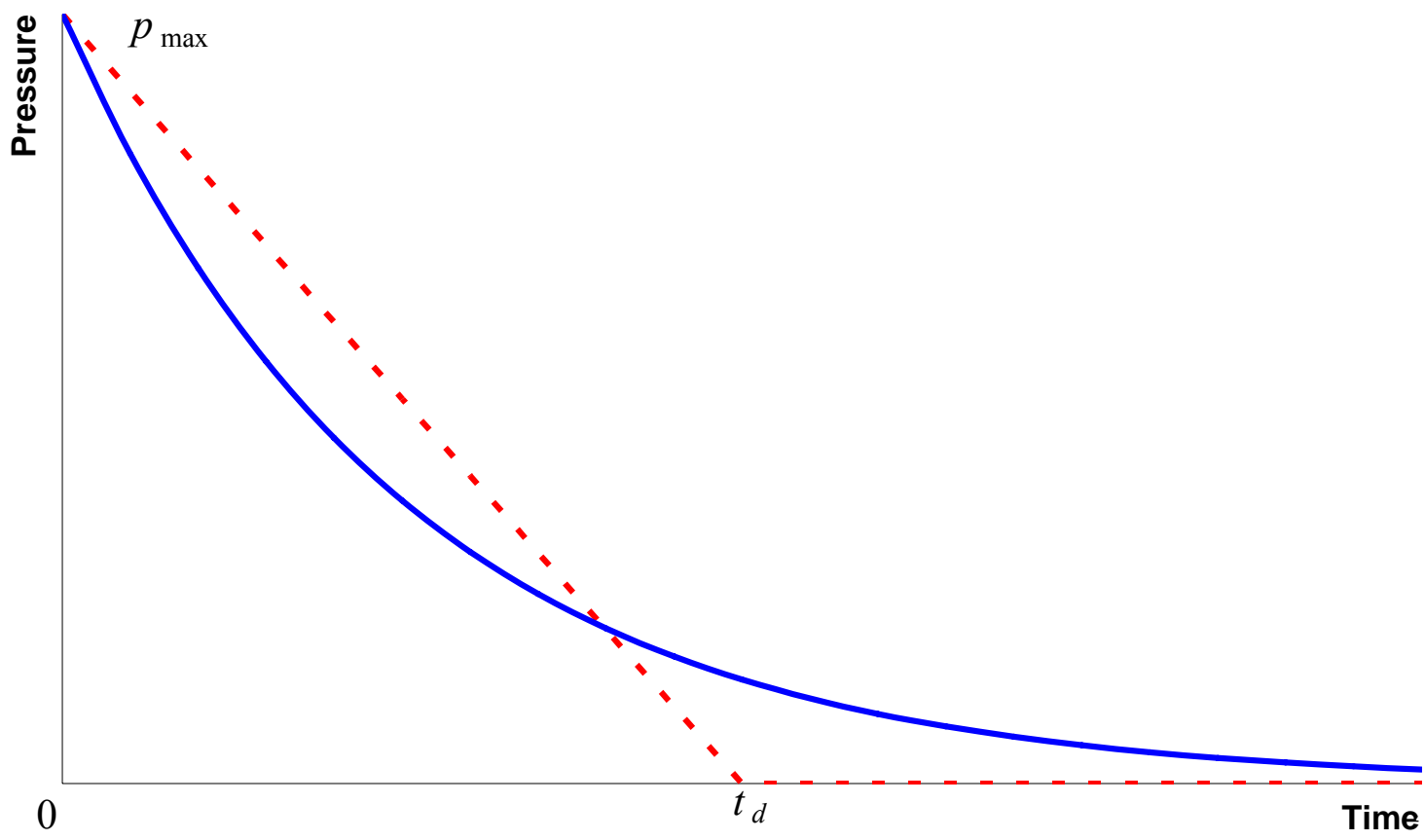

Figure 1 Pressure decay curves (blue/full - exponential, red/dotted - linear)

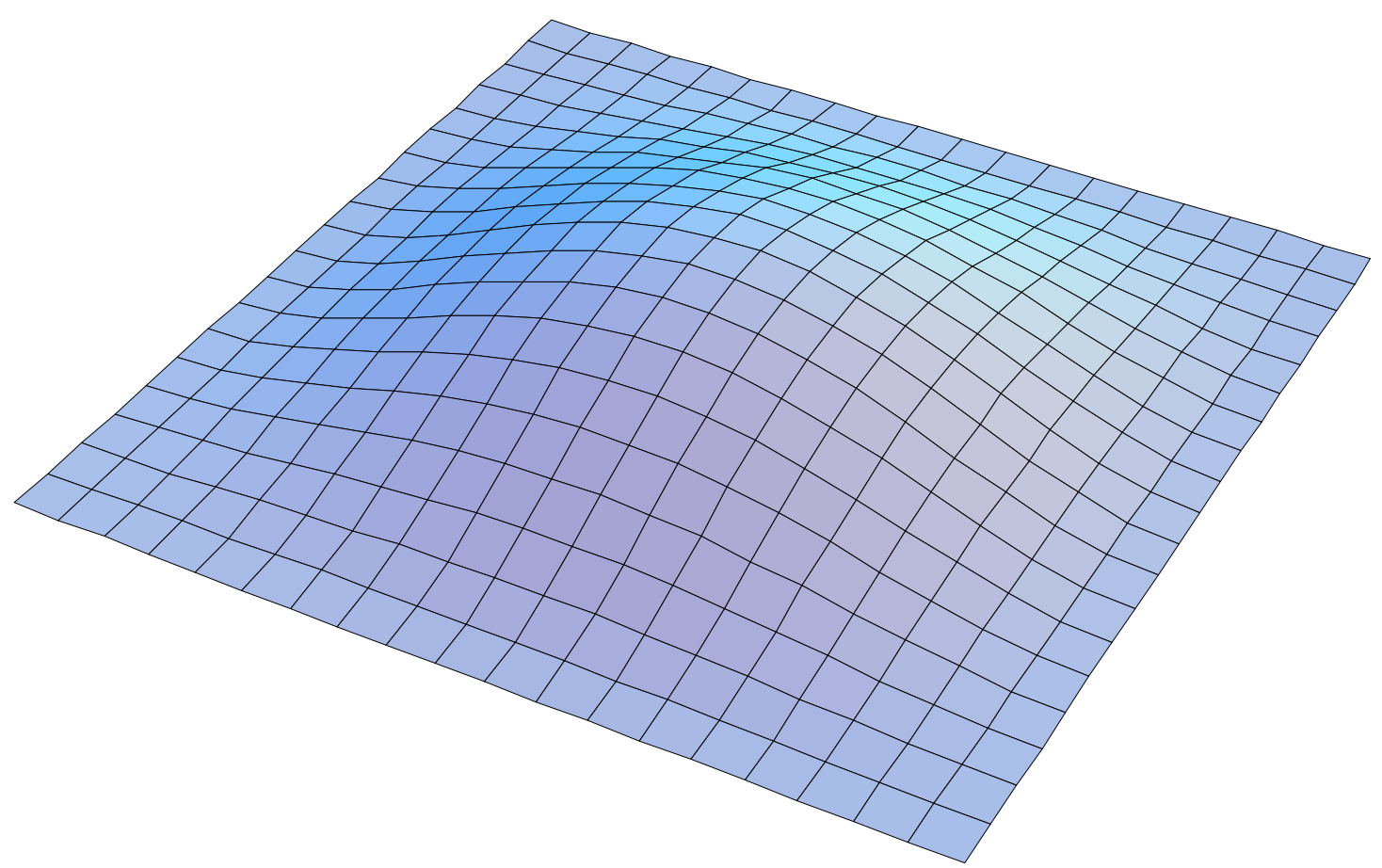

Figure 2 Deflections of the (square) plate 


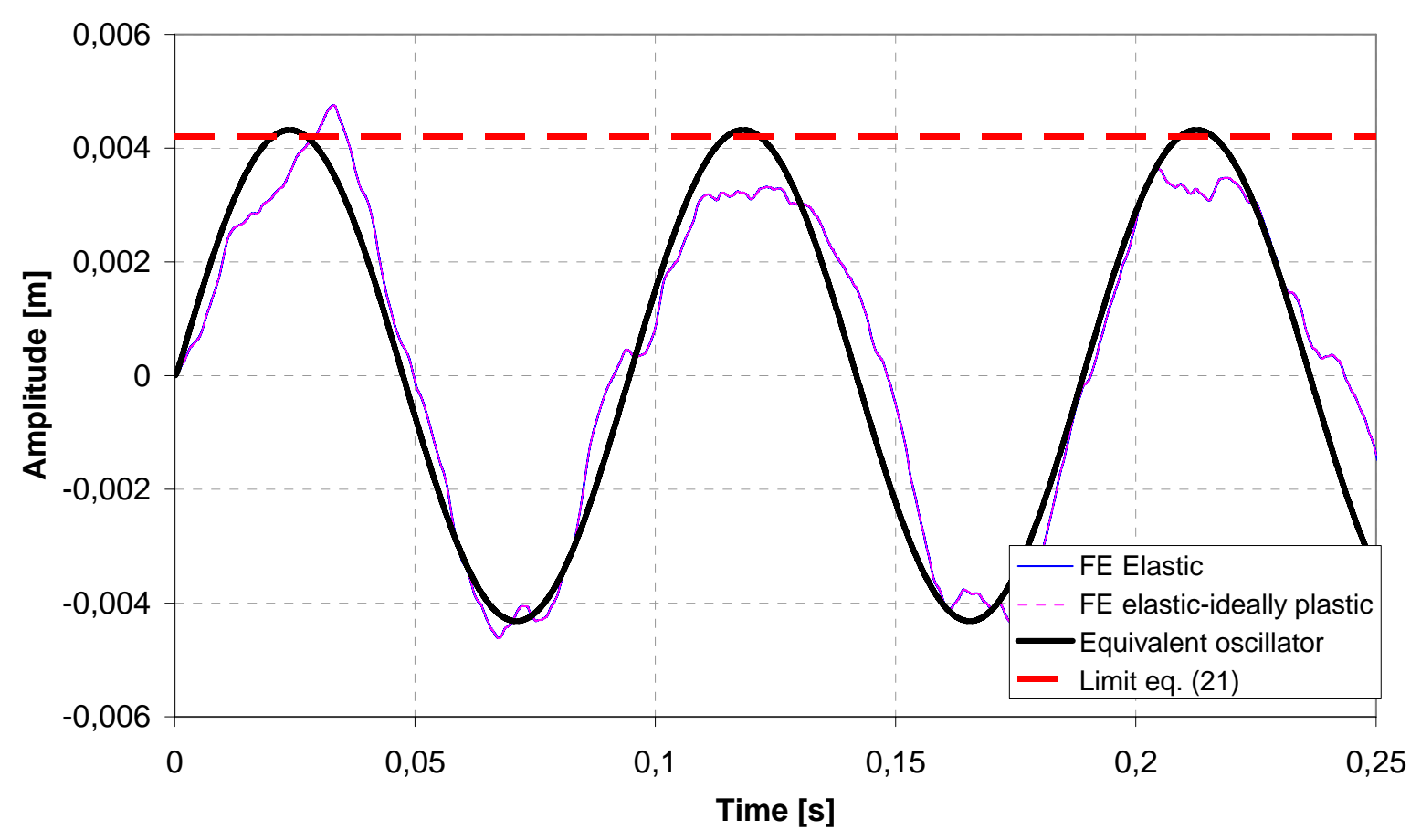

Figure 3 Deflections of the rectangular plate (very short overpressure phase $t_{d}=0,01 \mathrm{~T}$ )

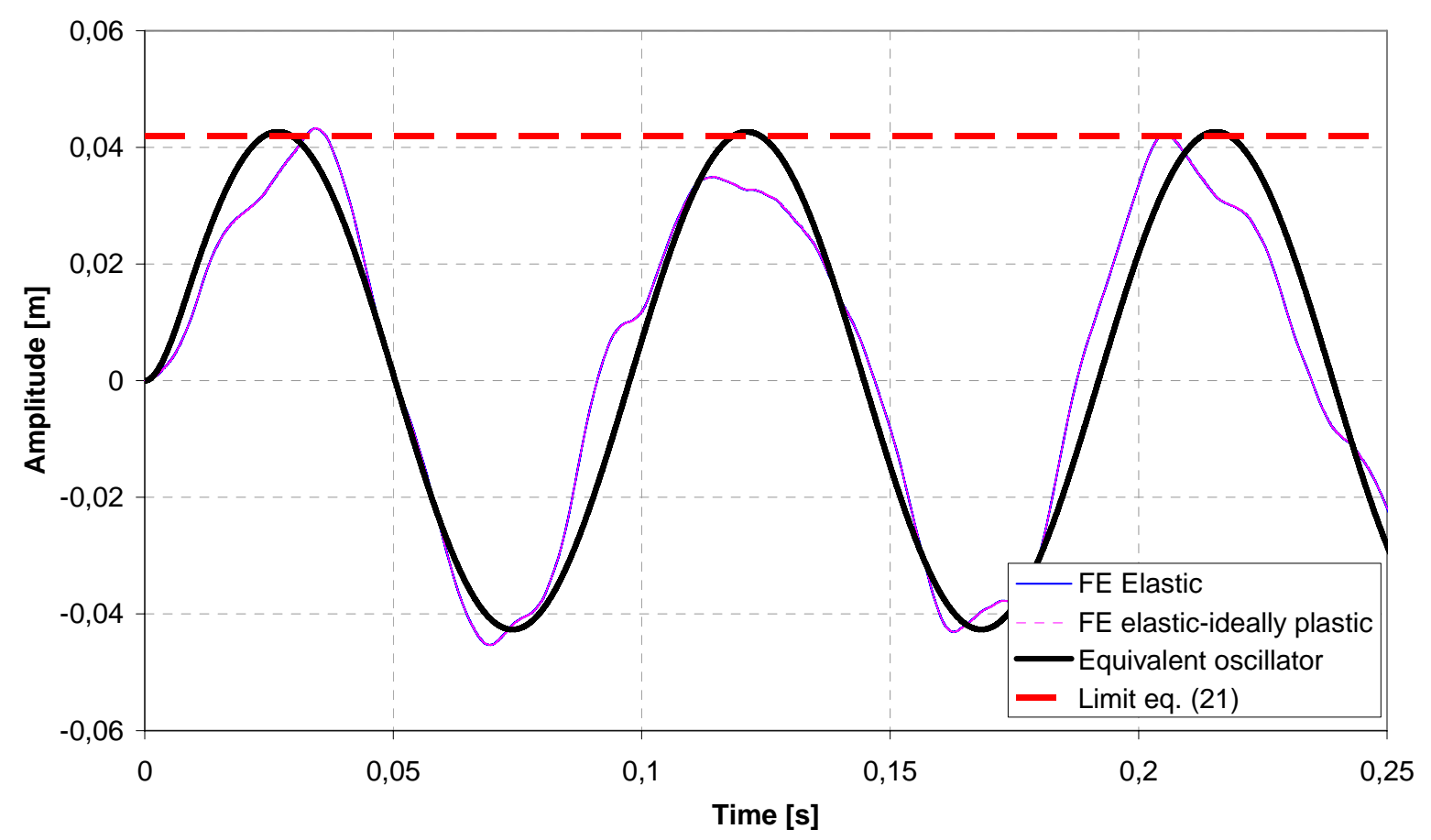

Figure 4 Deflections of the rectangular plate (short overpressure phase $t_{d}=0,1 T$ ) 


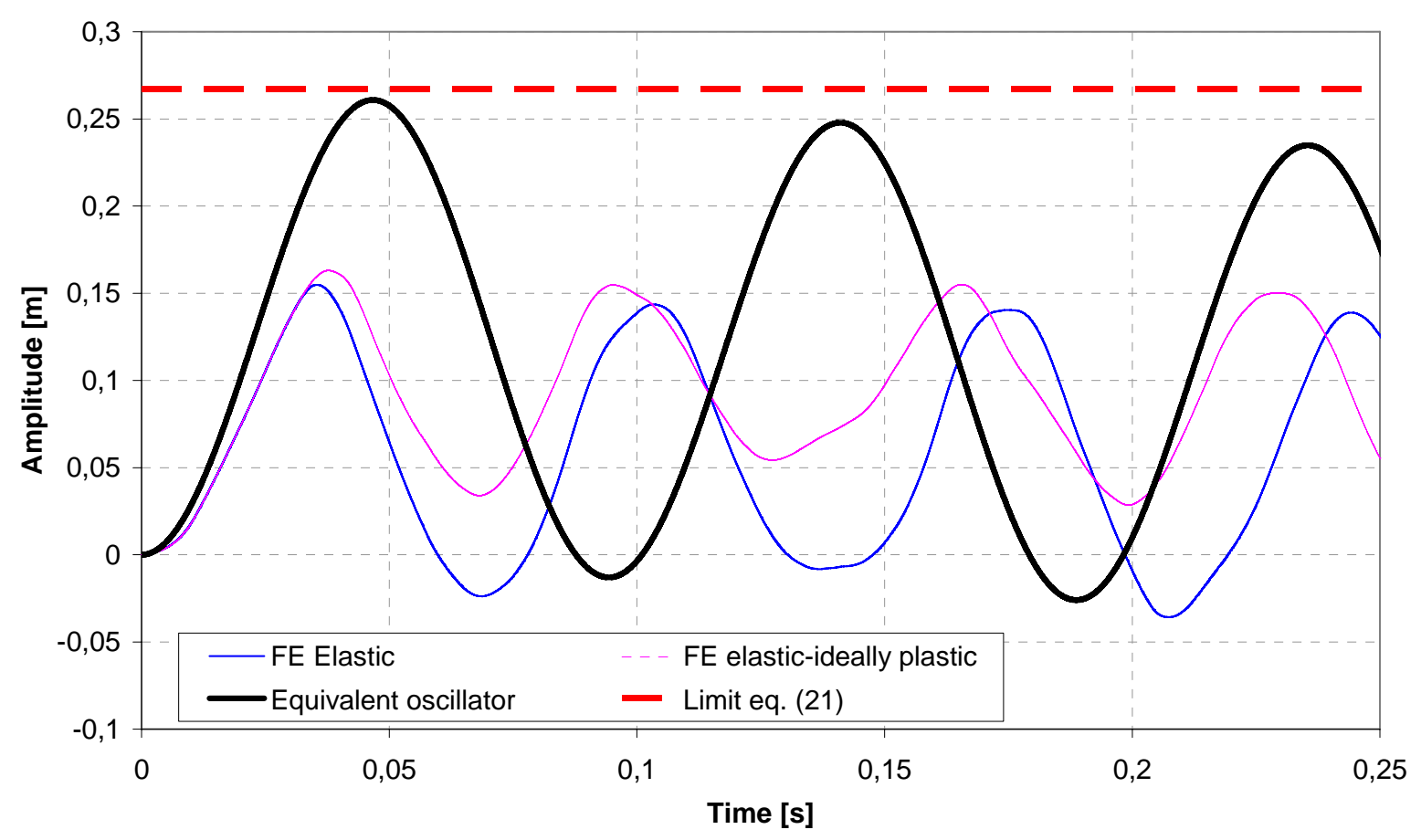

Figure 5 Deflections of the rectangular plate (long overpressure phase $t_{d}=10 \mathrm{~T}$ )

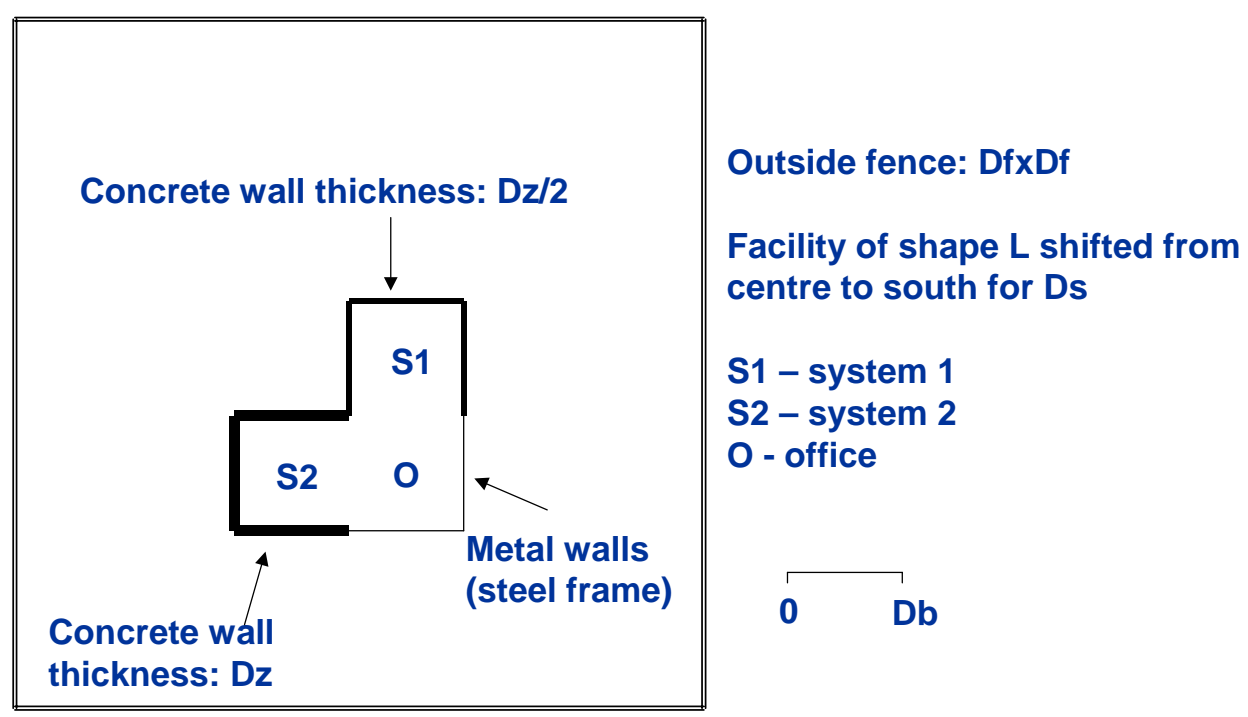

Figure 6 Ground Plan of the Example Facility with Building 


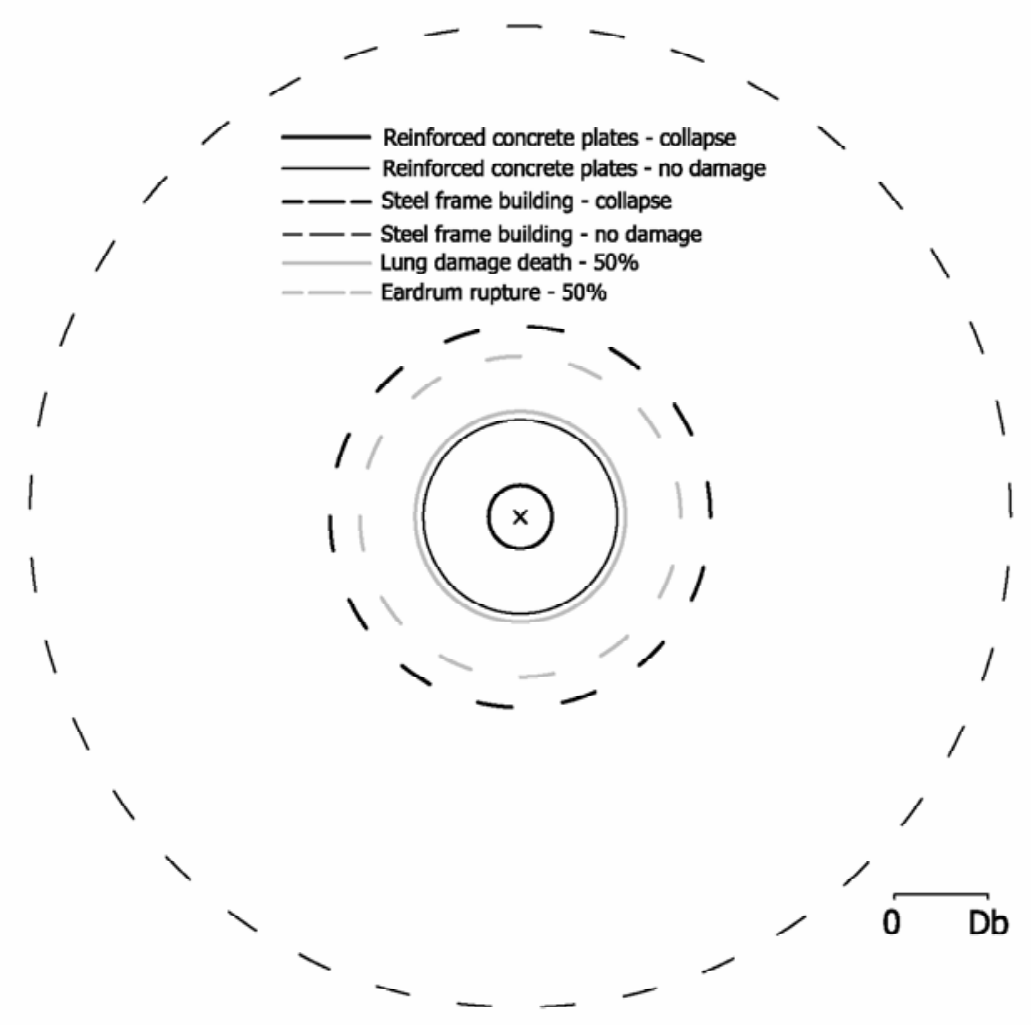

Figure 7 Stand-off distances around a selected bomb. The distances are not to scale

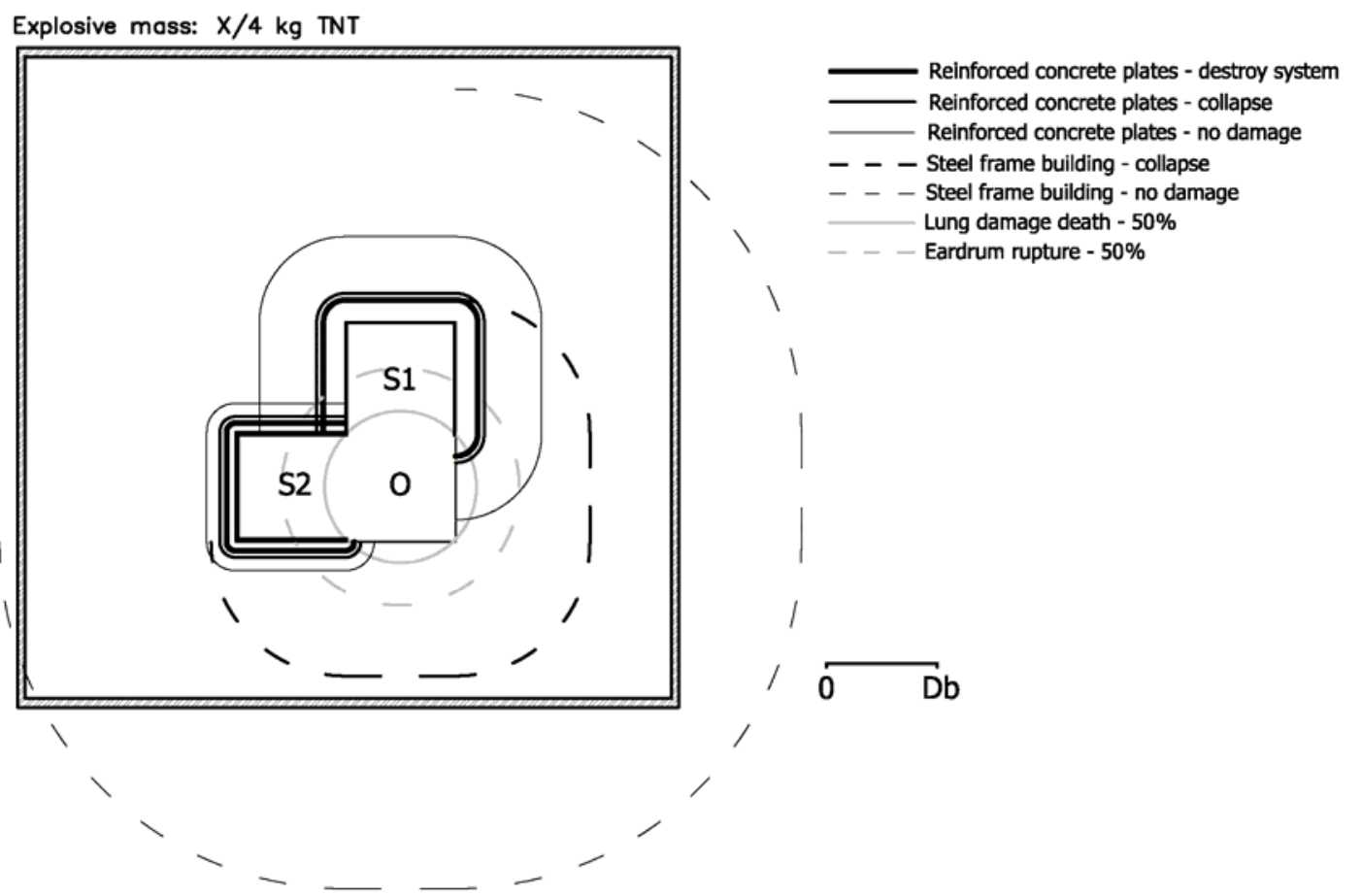

Figure 8 Stand-off distances for a selected bomb inside the controlled area. The distances are not to scale 


\section{TABLES}

Table 1 Shape factors (Harris \& Crede, 1976), (Beitz \& Küttner, 1981)

\begin{tabular}{|c|r|r|r|r|r|r|}
\hline $\boldsymbol{b} / \boldsymbol{a}$ & $\mathbf{1 . 0}$ & $\mathbf{1 . 5}$ & $\mathbf{2 . 0}$ & $\mathbf{2 . 5}$ & $\mathbf{3 . 0}$ & $\infty$ \\
\hline$C_{2}$ & 0.53 & 0.88 & 0.94 & & & \\
\hline$C_{3}$ & 0.225 & 0.394 & 0.431 & & & 0.455 \\
\hline$C_{5}$ & 1.24 & 1.82 & 1.92 & & & 2.0 \\
\hline$B_{a}$ & 35.98 & 24.57 & 23.77 & 23.77 & 23.19 & 22.37 \\
\hline$D$ & 0.0043 & 0.0111 & 0.0147 & & & \\
\hline
\end{tabular}

Table 2 Periods and overpressure durations

\begin{tabular}{|r|r|r|}
\hline $\begin{array}{c}\text { Overpressure } \\
\text { duration } \boldsymbol{t}_{\boldsymbol{d}}\end{array}$ & $\begin{array}{c}\text { Square plate } \\
\mathbf{T}=\mathbf{0 , 0 6 6 5} \mathrm{s}\end{array}$ & $\begin{array}{c}\text { Rectangular plate } \\
\boldsymbol{T}=\mathbf{0 , 0 9 6 7} \mathbf{~}\end{array}$ \\
\hline$t_{d}=0,01 T$ & $0,000665 \mathrm{~s}$ & $0,000967 \mathrm{~s}$ \\
\hline$t_{d}=0,1 \mathrm{~T}$ & $0,00665 \mathrm{~s}$ & $0,00967 \mathrm{~s}$ \\
\hline$t_{d}=1 T$ & $0,0665 \mathrm{~s}$ & $0,0967 \mathrm{~s}$ \\
\hline$t_{d}=10 T$ & $0,665 \mathrm{~s}$ & $0,967 \mathrm{~s}$ \\
\hline$t_{d}=100 T$ & $6,65 \mathrm{~s}$ & $9,67 \mathrm{~s}$ \\
\hline
\end{tabular}

